Contents List available at RAZI Publishing Acta Electronica Malaysia (AEM)

Journal Homepage: http://actaelectronicamalaysia.com

https://doi.org/10.26480/aem.01.2017.09.11

\title{
Design of Upright Intelligent Vehicle Based on Camera
}

\author{
Feng Jie, Liu Xiaojun \\ School of Electronic \& Information,Huanggang Normal University,Hubei Huanggang, China \\ *E-mail:18623582@QQ.com
}

This is an open access article distributed under the Creative Commons Attribution License, which permits unrestricted use, distribution, and reproduction in any medium, provided the original work is properly cited.

\begin{tabular}{ll}
\hline ARTICLE DETAILS & ABSTRACT \\
\cline { 1 - 2 } Article history: & $\begin{array}{l}\text { Design of mechanical and control system of walking uprighttype intelligent vehicle. The system uses MK60 as the } \\
\text { main controller, with the gyro sensor and acceleration sensor together, using complementary filter methods to } \\
\text { measure the til tangle and angular velocity of the intelligent vehicle. Using the PD algorithm to control the } \\
\text { Received 12 August 2016 }\end{array}$ \\
$\begin{array}{l}\text { Accepted 12 December 2016 } \\
\text { Available online 20 January 2017 }\end{array}$ & $\begin{array}{l}\text { MK60 array camera sensor, through a new kind of edge detection methods to complete the processing of image } \\
\text { information,attitude angle fusion of gyroscope and accelerometer using the complementary filtering } \\
\text { Keywords: }\end{array}$ \\
algorithm,complete the vertical,movement and steering control of the intelligent vehicle. After testing, the \\
intelligent vehicle to stay upright at the sametime, complete identification of the track, and runs smooth and fast.
\end{tabular}

control; PID algorithm; Path recognition

\section{INTRODUCTION}

Intelligent vehicle control technology has become an emerging technology, it involves automotive, electronic, automatic control, sensor technology, signal processing, knowledge fields of many disciplines. As an emerging technology, this technology provides the necessary technical support for the research of automobile master / passive safety, unmanned driving technology and other fields. Vertical running self balancing intelligent vehicle in abroad are widely used in automatic tracking logistics, patrol duty, base training aspects such as medium and short distance transport.From Segway in the United States to ChegCar in China, the two-wheeled self-balancing intelligent vehicle is moving into people's daily life. However, due to price and safety problems, it takes some time to popularize the application.

\section{SYSTEM ARCHITECTURE DESIGN}

The intelligent vehicle system uses Freescale's 32-bit controller KinetisK60 microcontroller as the core control processor,Kinetis has strong scalability, low power mixed signal microcontroller ARM CortexTMM4 kernel based, with high Real-time and so on.

This article uses the system structure shown in Fig.1. The camera OV7640 is used to collect information of the track; the photoelectric encoder is used to measure the distance traveled by the cart, according to the time of the running into the current speed of the car; the MPU3050 is a three-axis gyroscope, and the MMA8451 is an accelerometer that can be controlled by the fusion acceleration and angular velocity Car upright. These sensors to collect data through the bus to the K60 for processing, the microcontroller will be upright control, speed control and steering control together with the motor, the control car to complete the appropriate operation. K60 uses Bluetooth to communicate with the host computer for serial communication, which is convenient for checking the system faults and debugging parameters.The whole system is composed of Ni-Cd battery as power supply module.

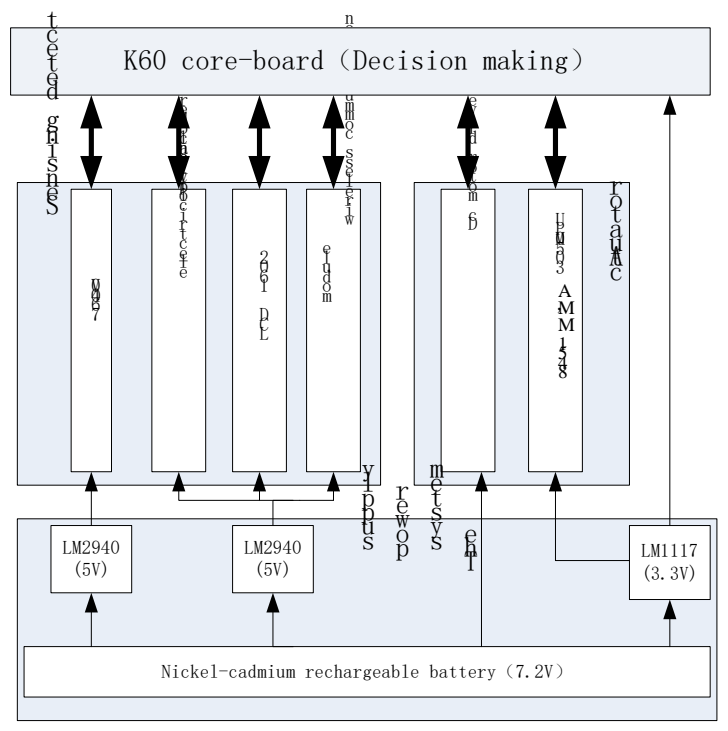

Fig. 1 The block diagram of system

\section{INTELLIGENT CAR HARDWARE CIRCUIT DESIGN}

\subsection{K60 SINGLE CHIP MINIMUM SYSTEM MODULE}

The control chip used in this paper is FREESCALE Kinetis K60 (referred to as K60) series, which is a 32-bit microcontroller, floating point operation, FLASH 512K, the paper designed K60 minimum system board with $150 \mathrm{M}$ standard frequency, through the test lock Phase ring up to $400 \mathrm{M}$, the bus frequency up to $200 \mathrm{M}$, can make the entire control system the best, the most stable play. At the same time K60 microcontroller also has the 
characteristics of low power consumption, reducing the overall system power supply burden. K60 minimum system includes 3.3V power supply, reset circuit, crystal oscillator circuit and I / 0 port and other components. The minimum system is necessary for the operation of the microcontroller, and the formation of the smallest system will not change, the design will K60 minimum system made of a separate module, easy to use and replace.

\subsection{POWER MODULE}

The hardware circuit power of the whole vehicle is supplied by rechargeable Ni-cd battery $(7.2 \mathrm{~V}, 2000 \mathrm{mAh})$. But the system of the various circuit modules required by the operating voltage and operating current vary, so a variety of voltage stabilizing circuits are designed to convert the battery voltage to the voltage needed by each module. The motor drive circuit requires $7.2 \mathrm{v}$ voltage, which is provided directly with the battery. Microcontroller, SD card and gyro module require $3.3 \mathrm{~V}$ voltage, photoelectric encoder, camera, display and Bluetooth serial module requires $5 \mathrm{~V}$ power supply. $5 \mathrm{~V}$ from the LM2940, 3.3V voltage provided by the LM1117.

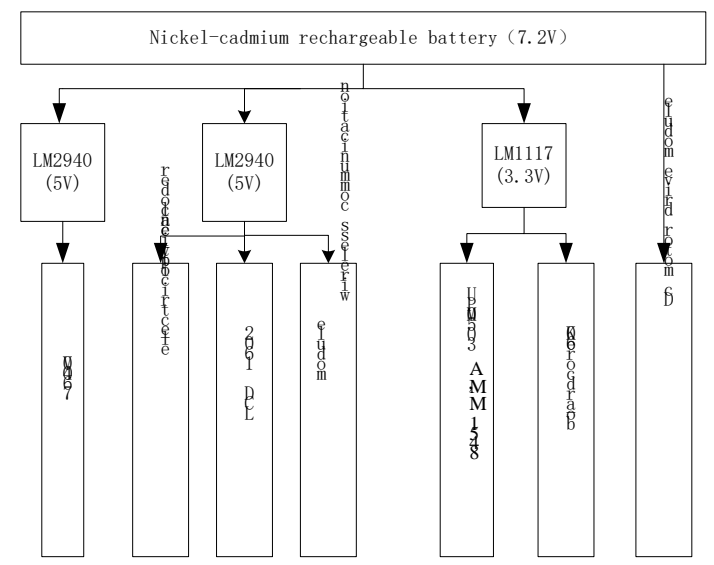

Fig.2 Power supply diagram

\subsection{MOTOR DRIVE MODULE}

In order to improve the response speed of the servo motor, the servo motor of the upright driving intelligent vehicle uses the $7.2 \mathrm{~V} 2000$ $\mathrm{mAh} \mathrm{Ni}-\mathrm{Cd}$ battery to supply power directly. Fig.3 for the motor control schematic. Motor drive program using four BTS7960 constitute two full-bridge circuit, respectively controlling two motor forward and reverse.

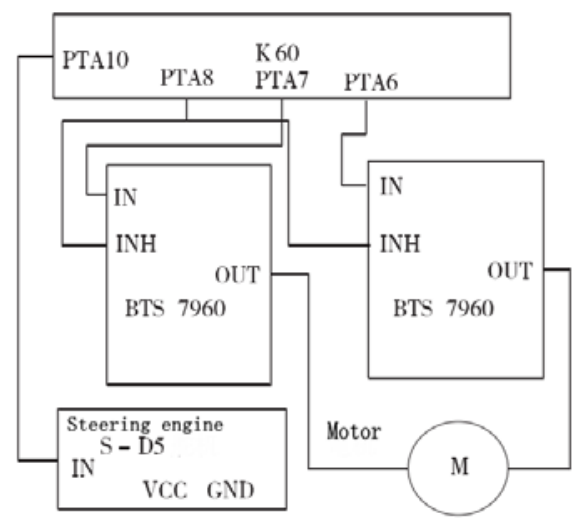

Fig.3 Hardware circuit diagram of the execute module

\subsection{GYROSCOPES AND ACCELEROMETERS}

Accelerometer and gyroscope is the necessary information feedback device, this article is selected by Freescale's MMA8451 three-axis acceleration sensor and MPU3050 gyroscope. Schematic diagram shown in Fig.4.

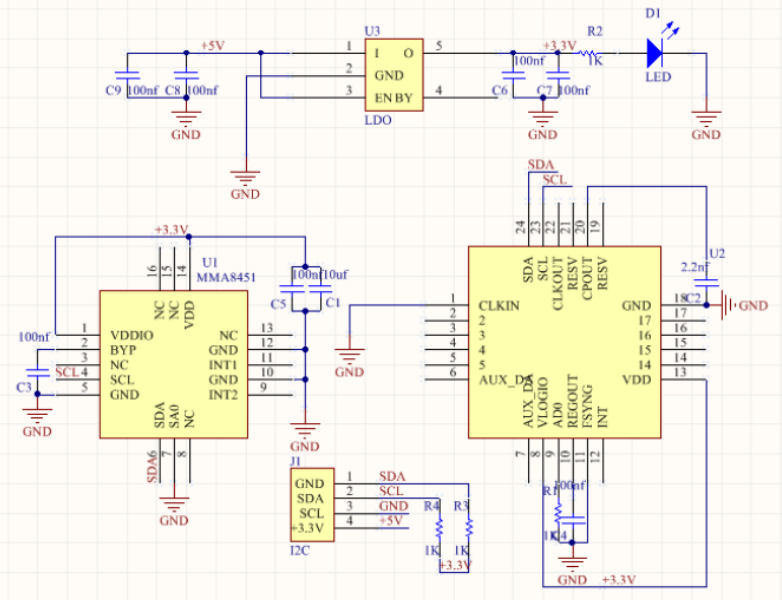

Fig.4 The accelerometer and gyroscope circuit

\section{DESIGN AND IMPLEMENTATION OF SOFTWARE SYSTEM}

\subsection{GENERAL DESIGN OF SOFTWARE SYSTEM}

The main program is only responsible for initialization and data transmission, LCD display and other tasks, in the timer interrupt in the sensor data acquisition, control the amount of control and control output and other operations. The main functions of the software include:(1) the sensor signal acquisition, processing; (2) motor PWM output; (3) car mold operation control: upright control, speed control, direction control; (4) car mold operation process control: program initialization, car mode start and end, Car model status monitoring; (5) car model information display and parameter settings: status display, host computer monitoring, parameter settings, etc.The first class includes 1-3 functions, which belong to the need for precise time period execution, and thus can be done in a periodic timed interrupt. The second category includes the 4-5 function. Its execution does not require an accurate time period. System software flow chart shown in Fig.5.

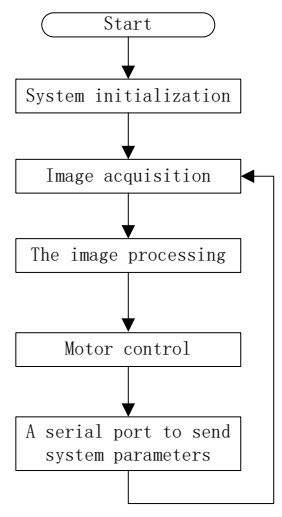

\subsection{UPRIGHT CONTROL OF INTELLIGENT VEHICLE}

Upright walking car is equivalent to the inverted pendulum, it is not like the general pendulum, like their own back to the equilibrium position, because the inverted pendulum of the return force and deflection direction of the same direction, not only will not make the inverted pendulum back to balance Position, but will be more and more deviated from the equilibrium position, so to make the inverted pendulum to maintain balance, must be applied with a deflection of the opposite direction of the external force [6]. Combined with the car may be the direction of movement, only through the car before and after the acceleration of the acceleration to combat the tendency to tilt it. To generate the appropriate acceleration, first through the gyroscope to collect the car around the axle speed of the car, the accelerometer collected perpendicular to the body of the acceleration, after conversion unit, let the angular velocity integral approximation acceleration calculated angle and angular velocity through the complementary filtering algorithm fusion, this Angle is the calculation of the node relative to the previous calculation of the angle of change in the node, through the PD operation can be used as a control motor PWM value, the motor to the appropriate speed of operation, to control the requirements of car balance. 
4.3 IMAGE PROCESSING MODULE

In the process of driving the model car, the master based on the edge of the track to track, so the edge of the detection is particularly important. The runway background is dark blue, the runway is a smooth white plate, the edge of which is black electrical tape. The runway image collected by the camera is a grayscale image, and for the runway edge of this article, the white track plate is split with the black runway edge.

According to the characteristics of the track image, this paper adopts a simple and efficient new edge detection algorithm. A detailed description is: Row as a unit of processing,the edge operator is selected by using the edge operator $[1,-1]$ for the neighborhood convolution operation, where the maximum value is the edge of the row. In order to improve the efficiency of the operation and weaken the influence of the interference signal, the edge of the next row is determined by the position of the edge of the previous line after the edge of the first row is obtained. The specific steps are as follows:

1)The convolution operation is performed by the edge operator [1, -1] from both the center of the first row of the first image.Scan from center to left.

2)The first line of the image is convoluted by the edge operator [1, $-1]$.

3)Select the point of the maximum value in the convolution operation result, that is, the edge point of the row.

4)After obtaining the runway edge of the first row, in order to reduce the edge extraction time and increase the speed of operation, the second line and the subsequent lines are used to track the edge line acquisition, as shown in Fig.6.

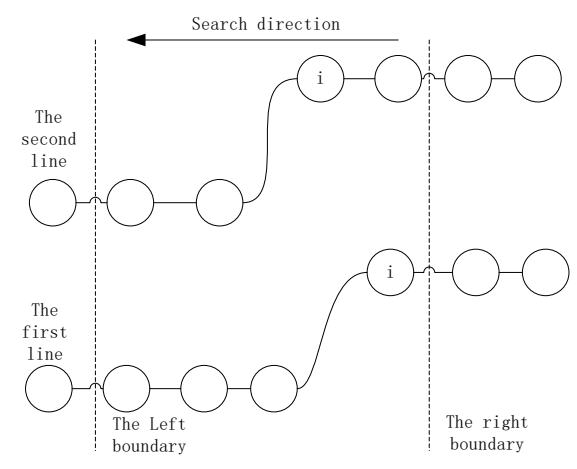

Fig.6 The diagram of tracking search boundary

After determining the first line edge points, respectively to the left and right several pixels as boundary patrol second line, second line starts line does not need to start from the centre of convolution, but from the first line given line starting point convolution. Using the method of tracking line can reduce the line cycle of each line and improve the edge detection, but if the line is too small, the line may be lost.

\subsection{INTELLIGENT CAR STEERING CONTROL}

According to the degree of deviation from the central location of the car to the car to allocate different sizes of steering value, the basic idea is the greater the degree of deviation to the car the greater the value of the steering, which can ensure that the deviation from the track center in the case of the car to the maximum steering value to ensure that the car quickly back to the center position, the algorithm is used in the PD control: steering PWM value calculation formula:

$P W M=K p \quad e(t)+K d[e(t)-2 e(t-1)+e(t-2)]$ Simple PD control in the car in the low-speed state when the effect can be, with the speed of the car in the steering angle of the angular speed should be increased accordingly, in the face of continuous steering steering, due to inertia, steering time is serious Delayed the phenomenon, causing the car out of the track. In order to solve this problem, the system also measured the angular velocity of the normal rotation around the track plane. Similarly, the PD algorithm calculates a gain to offset the steering delay due to inertia [7]. By constantly adjusting the size of the PD value and observe the phenomenon, to obtain the appropriate PD value, the more perfect solution to the problem of steering delay.

\subsection{INTELLIGENT VEHICLE SPEED CONTROL}

Using the pulse capture port on the microcontroller to capture the vehicle speed sensor in the unit time pulse to obtain the current speed, PI closed-loop control, according to the actual load conditions and rapid adjustment of the PWM wave to adjust the torque in time, and ultimately stabilize the car And in order to avoid the interference of the speed control to the upright control, the sum of the 10 main cycle periods is measured when measuring the motor speed, and the output of the PI control is divided by 10 and the output is 10 times. The incremental PID calculation The formula is

$$
\text { Kpe }(t)+K i[e(t)-e(t-1)](2)
$$

In the ordinary track, PI control is sufficient to meet the requirements, but in the passage of about $10^{\circ}$ ramp, the simple PI control can not produce enough acceleration car, and downhill can not decelerate in time, which is not conducive to fast and smooth Complete the game. In order to solve this problem, in the car uphill to improve the zero deviation of the accelerometer, so that it has a tendency to tilt forward, and downhill to reduce the zero deviation of the accelerometer, so that it has a tendency to tilt backward Produce enough acceleration to accelerate and decelerate. In addition, on the ordinary track, the speed deviation of the car is also limited, more than the limit is to increase or decrease the zero deviation of the accelerometer, so that the corresponding direction of tilt, resulting in an additional acceleration.

\section{CONCLUSION}

In this paper, the use of gyroscope, acceleration sensor and camera to collect external signals, through the vertical control, speed control and direction control to achieve intelligent car upright tracking function. The intelligent vehicle stability, speed of $2 \mathrm{~m} / \mathrm{s}$, can realize a variety of track types of identification and tracking, to achieve the desired effect.

\section{ACKNOWLEDGMENT}

The project was supported by the project of Huanggang Normal University (Grant No. zxfz2016A014).

\section{REFERENCES}

[1] MILLER R M, SHAH S L, WOOD R K, etal. Forecast and PID control technology [J]. ISA Transactions, 2008,38(1):11-23.

[2] NORMEY-RICOJE, GOJ, CAMACHOEF. A Smith-predictorbased generalized predictive controller for mobile robile pathtracking[]].Control Engineering Practice, 2008,16(4):407-428.

[3] DN Mpcec. Freescale Semiconductor Technical Data. [J]. Eetimes Com,2013,04:23-28.

[4] B Remenant. Sequencing of K60, type strain of the major plant pathogen Ralstonia solanacearum[J]. Journal of Bacteriology, 2012, 194(10):2742-3.

[5] Zhao Lianna, Zhao Kai, Li Lei, Zhao Xiujuan,Li Yang. Research on Special Path Recognition and Control Algorithm Based on K60 for Intelligent vehicle [J]. Electronic Technology (Chinese), 2016, 53 (3), 20-23.

[6] YU Shaodong, HUANG Danping, TIAN Jianping, HU Yong. Control System Design of Intelligent vehicle Based on Kinetis K60[J]. Journal of Sichuan University of Science \& Engineering(Natural Science Edition,Chinese), 2014, 27(5), 37-42.

[7] GAO Zheng-zhong, ZHAO Li-na, LI Shi-guang, BAI Xingzhen, SONG Sen-sen. Design of Intelligent Vehicle Control System Based on Camera[J]. Automation \& Instrumentation (Chinese), 2015, 32(6), 1-5.

[8] Yang Zhengcai, Lü Ke, Zhu Le. Design of Upright-moving Intelligent vehicle Based on 32 -bit Kinetis - K60 Microcontroller of Freescale[J]. Journal of Hubei University of Automotive Technology (Chinese) , 2014, 28(2), 46-51.

[9] XU Wei, CHEN Wanmi.Design of a Predictive Adjustable Two Wheel Upright Intelligent Car[J]. Industrial control computer (Chinese) , 2016, 29(8), 45-49.

[10] QIN Gang, DU Chao, WU Danyi. Simulation and Analysis on Intelligent CarTurning Control Algorithm[J]. Techniques of Automation \& Applications（Chinese）, 2012, 31(12), 49-64. 